\title{
Wide-View MVA-LCDs With an Achromatic Dark State
}

\author{
Meizi Jiao, Zhibing Ge, Student Member, IEEE, and Shin-Tson Wu, Fellow, IEEE
}

\begin{abstract}
A multi-domain vertical alignment liquid crystal display (MVA LCD) using a negative $\mathrm{C}$-plate and a biaxial film is proposed to obtain an achromatic dark state. In comparison to conventional approach which uses a negative $C$-plate and a positive A-plate, the maximum light leakage over entire viewing cone is reduced by $\sim 6 X$ at $\lambda=450 \mathrm{~nm}$ and $\sim 4 X$ at $\lambda=650 \mathrm{~nm}$. The achromatic dark state is essential for improving the contrast ratio, viewing angle and color saturation of MVA LCDs.
\end{abstract}

Index Terms-Biaxial film, liquid crystal display, vertical alignment, wide view.

\section{INTRODUCTION}

$\mathbf{M}$ ULTI-DOMAIN vertical alignment (MVA) is widely used for wide-view liquid crystal display (LCD) TVs. It exhibits a high contrast ratio (CR) at normal viewing angle. To suppress dark state light leakage at oblique angles and thereby obtain a wide viewing angle, several phase compensation schemes using uniaxial films and biaxial films have been proposed. [1]-[4] The 100:1 isocontrast contour at an optimized wavelength (usually $\lambda \sim 550 \mathrm{~nm}$ ) can cover the entire viewing cone, however, the light leakage at other wavelengths are not optimized. As a result, the light leakages from blue and red colors significantly degrade the CR and color saturation. Several approaches have been proposed to obtain achromatic dark state [5], [6]; however, these approaches either involve too many layers of compensation films or require compensation films with an abnormal dispersion (i.e., refractive index increases with wavelength).

In this paper, we propose a new compensation scheme for MVA-LCDs to achieve achromatic dark state for improving $\mathrm{CR}$ and viewing angle under white light illumination. Our compensation scheme requires only two compensation films with normal dispersion: one negative C-plate and one biaxial film. The dispersion of liquid crystal (LC) usually follows the Cauchy model while the dispersion of compensation films can be much smaller. [7] This difference has advantage in our configuration to balance the effective phase retardation at different wavelengths. With the help of Poincaré sphere [8], the phase parameters of the compensation films are optimized

Manuscript received January 27, 2009. Current version published April 01, 2009. This work was supported by Chi-Mei Optoelectronics Corporation (Taiwan).

The authors are with the College of Optics and Photonics, University of Central Florida, Orlando, FL 32816 USA (e-mail: mjiao@mail.ucf.edu, zge@creol. ucf.edu, swu@mail.ucf.edu).

Color versions of one or more of the figures in this paper are available online at http://ieeexplore.ieee.org.

Digital Object Identifier 10.1109/JDT.2009.2015685

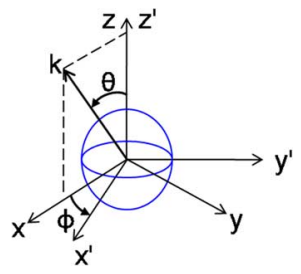

Fig. 1. Refractive index ellipsoid and propagation direction in principal coordinate system and local coordinate system.

at $\lambda=550 \mathrm{~nm}$. Furthermore, within a realistic range, their dispersions are optimized at 450 and $650 \mathrm{~nm}$ to suppress the light leakage over a wide spectral range. The light leakage in the whole visible spectrum is significantly reduced as compared to the conventional compensation scheme comprised of a negative C-plate and a positive A-plate. The maximum global light leakage is reduced by $\sim 6 X$ at $\lambda=450 \mathrm{~nm}$ and $\sim 4 X$ at $\lambda=650 \mathrm{~nm}$.

\section{DESIGN CONCEPTS}

\section{A. Optical Calculation of Biaxial Film}

The biaxial films used in LCD compensation are usually fabricated by mechanically stretching or compressing method. The optic axis is perpendicular or parallel to the stretching or compressing direction. Therefore, the $z$-axis of the principal coordinates $\left(x^{\prime}, y^{\prime}, z^{\prime}\right)$ of the biaxial medium is still perpendicular to the film surface. Without losing the generality, we choose an $(x, y, z)$ coordinate system that the incident light is located in $x-z$ plane. The refractive index ellipsoid and the incident beam can be illustrated in Fig. 1. Here $x^{\prime}, y^{\prime}$ and $z^{\prime}$ are three principal coordinates of the biaxial film. The $x-y$ plane is parallel to the biaxial film surface. The $z$-axis is coincident with $z^{\prime}$. In this case, the dielectric tensor of biaxial film can be obtained by doing the Eulerian angle transformation from $\left(x^{\prime}, y^{\prime}, z^{\prime}\right)$ principal coordinates system to the $(x, y, z)$ coordinate system.

$$
\begin{aligned}
\bar{\varepsilon}= & {\left[\begin{array}{lll}
\varepsilon_{x x} & \varepsilon_{x y} & \varepsilon_{x z} \\
\varepsilon_{y x} & \varepsilon_{y y} & \varepsilon_{y z} \\
\varepsilon_{z x} & \varepsilon_{z y} & \varepsilon_{z z}
\end{array}\right] } \\
\bar{\varepsilon}= & {\left[\begin{array}{ccc}
\cos \phi & -\sin \phi & 0 \\
\sin \phi & \cos \phi & 0 \\
0 & 0 & 1
\end{array}\right]\left[\begin{array}{ccc}
n_{x^{\prime}}^{2} & 0 & 0 \\
0 & n_{y^{\prime}}^{2} & 0 \\
0 & 0 & n_{z^{\prime}}^{2}
\end{array}\right] } \\
& \times\left[\begin{array}{ccc}
\cos \phi & \sin \phi & 0 \\
-\sin \phi & \cos \phi & 0 \\
0 & 0 & 1
\end{array}\right]
\end{aligned}
$$


where

$$
\begin{aligned}
& \varepsilon_{x x}=n_{x^{\prime}}^{2} \cos ^{2} \phi+n_{y^{\prime}}^{2} \sin ^{2} \phi \\
& \varepsilon_{y y}=n_{x^{\prime}}^{2} \sin ^{2} \phi+n_{y^{\prime}}^{2} \cos ^{2} \phi \\
& \varepsilon_{z z}=n_{z^{\prime}}^{2} \\
& \varepsilon_{x y}=\varepsilon_{y x}=\left(n_{x^{\prime}}^{2}-n_{y^{\prime}}^{2}\right) \sin \phi \cos \phi \\
& \varepsilon_{x z}=\varepsilon_{z x}=0 \\
& \varepsilon_{y z}=\varepsilon_{z y}=0 .
\end{aligned}
$$

Once the dielectric tensor of biaxial film is obtained, the optical properties can be calculated using the extended $2 \times 2$ matrix method.

From Maxell's equation, a matrix representation of electric filed and magnetic field of the propagating light can be derived.

$$
\frac{\partial}{\partial z}\left[\begin{array}{c}
E_{x} \\
E_{y} \\
\hat{H}_{x} \\
\hat{H}_{y}
\end{array}\right]=i k_{0} \mathbf{Q}\left[\begin{array}{c}
E_{x} \\
E_{y} \\
\hat{H}_{x} \\
\hat{H}_{y}
\end{array}\right]
$$

where

$$
\begin{aligned}
& \hat{\mathbf{H}}=\left(\frac{\mu_{0}}{\varepsilon_{0}}\right)^{1 / 2} \mathbf{H} \\
& \mathrm{Q}= \\
& {\left[\begin{array}{cccc}
-\frac{\varepsilon_{z x}}{\varepsilon_{z z}} \sin \theta & -\frac{\varepsilon_{z y}}{\varepsilon_{z z}} \sin \theta & 0 & 1-\frac{1}{\varepsilon_{z z}} \sin ^{2} \theta \\
0 & 0 & -1 & 0 \\
-\varepsilon_{y x}+\varepsilon_{y z} \frac{\varepsilon_{z x}}{\varepsilon_{z z}} & -\varepsilon_{y y}+\varepsilon_{y z} \frac{\varepsilon_{z y}}{\varepsilon_{z z}} \sin ^{2} \theta & 0 & \frac{\varepsilon_{y z}}{\varepsilon_{z z}} \sin \theta \\
\varepsilon_{x x}-\varepsilon_{x z} \frac{\varepsilon_{z x}}{\varepsilon_{z z}} & \varepsilon_{x y}-\varepsilon_{x z} \frac{\varepsilon_{z y}}{\varepsilon_{z z}} & 0 & -\frac{\varepsilon_{x z}}{\varepsilon_{z z}} \sin \theta
\end{array}\right]}
\end{aligned}
$$

Implementing dielectric tensor values of biaxial film as in (1) into the $\mathbf{Q}$ matrix in (2), we can extend the same method developed for uniaxial films to calculating the optical properties for biaxial films. Our calculations follow the backward-eigenwave method described by Ge [9]. Detailed derivation and formulation of backward-eigenwave method can be found in [9].

\section{B. Single Wavelength Wide-View Configuration}

Fig. 2(a) shows a commonly used compensation scheme for MVA-LCDs using one positive A-plate and one negative C-plate. The maximum light leakage of a MVA cell occurs when viewed from the bisector direction of the crossed polarizers. If the light leakage at all bisectors can be suppressed, then the viewing angle of the LCD can be significantly enhanced. Therefore, we optimized the compensation films by suppressing the light leakage at $\theta=70^{\circ}$ and $\varphi=45^{\circ}$, where $\theta$ is polar angle and $\varphi$ is azimuthal angle. The polarization state of the light traveling through all optical layers is traced on Poincaré sphere, as shown in Fig. 2(b).

The MVA LC cell is sandwiched between two crossed linear polarizers. When viewed from an oblique angle, the absorption axes of the polarizer and analyzer are no longer perpendicular to each other resulting in a deviation between the transmission

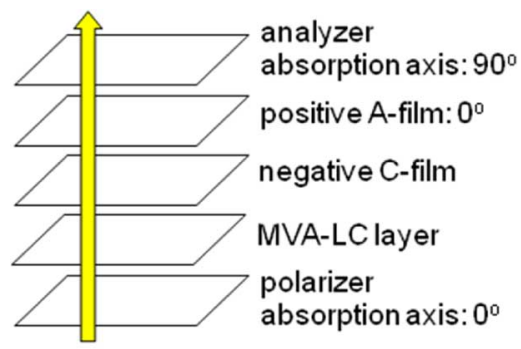

(a)

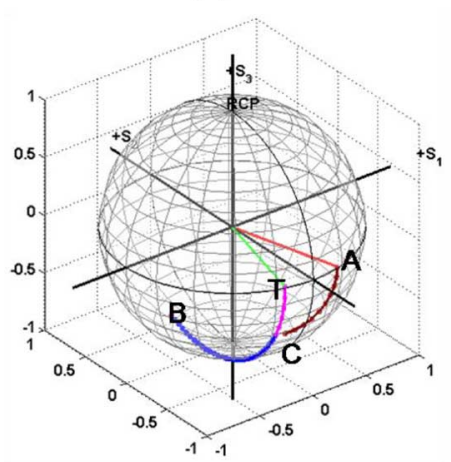

(b)

Fig. 2. (a) Single wavelength wide view MVA configuration with one negative C-plate and one positive A-plate. (b) Poincaré sphere representation of the compensation principle of the configuration in (a).

axis of the polarizer and the absorption axis of the analyzer, represented by point $\mathrm{T}$ and point $\mathrm{A}$ in Fig. 2(b). After the backlight passing through polarizer, it is converted to linearly polarized light, with its polarization state located at point T. After the LC layer, the polarization state is moved from point $\mathrm{T}$ to point $\mathrm{B}$. Then negative C-plate moves the polarization state back to point C. The LC and negative C-plate together behave like an effective positive C-plate. Finally, positive A-plate converts the polarization state from point $\mathrm{C}$ to point $\mathrm{A}$, which is the absorption axis of the analyzer. Here the positive A-plate should be approximately a quarter-wave plate and the optic axis should be the same as the absorption axis of the bottom polarizer.

Without compensation films, the viewing angle of MVA is very narrow. The $\mathrm{CR} \geq 100: 1$ is limited to $20^{\circ}$ viewing cone. With A-plate and C-plate compensation, the viewing angle of MVA is significantly enhanced (the CR $\geq 100: 1$ is over $80^{\circ}$ viewing cone).

However, this compensation is usually optimized at a single wavelength, $\lambda \sim 550 \mathrm{~nm}$; the light leakage at other wavelengths is ignored. Fig. 3 shows the Poincaré sphere tracing and dark state light leakage of the configuration shown in Fig. 2(a) for both the red light and blue light. As shown in Fig. 3(a) (for $\lambda=450 \mathrm{~nm}$ ) and Fig. 3(c) (for $\lambda=650 \mathrm{~nm}$ ), the final polarization state is located at point $\mathrm{D}$. Point $\mathrm{D}$ is departed from the absorption axis of analyzer, point A. Light leakage occurs for both cases. Angular light leakage is depicted in Fig. 3(b) (for $\lambda=$ $450 \mathrm{~nm}$ ) and (d) (for $\lambda=650 \mathrm{~nm}$ ), in which the light leakage is normalized to the maximum transmittance of two parallel linear polarizers. Two factors cause the light leakage at wavelengths other than the optimized one: 1) the effective phase is inversely proportional to the wavelength, and 2) the birefringence 


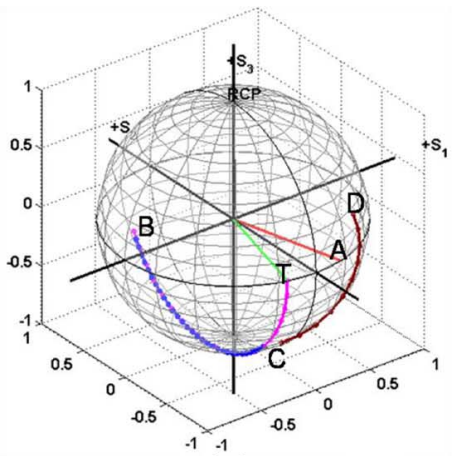

(a)

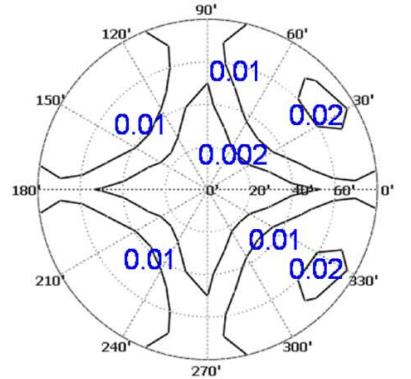

(b)

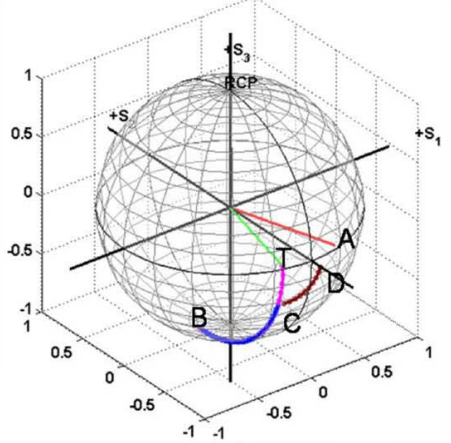

(c)

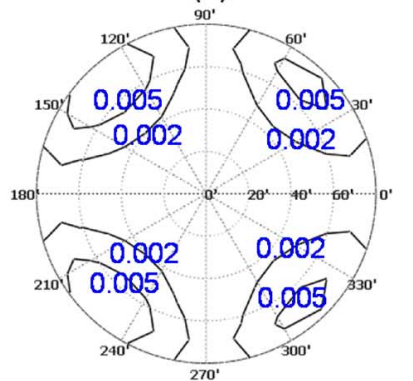

(d)

Fig. 3. Polarization state tracing on Poincaré sphere of the conventional compensation scheme at (a) $\lambda=450 \mathrm{~nm}$ and (c) $\lambda=650 \mathrm{~nm}$, and (b) and (d) are the corresponding calculated angular light leakage.

decreases with wavelength in the normal dispersion range. The arc length on Poincaré sphere depends on the phase retardation of the corresponding optical layer. Therefore, the arc length is shorter for a longer wavelength. From the tracing on Poincaré sphere, the dispersion of different layers cannot be compensated by each other under this compensation configuration.

\section{Broadband Wide-View Configuration}

To suppress the light leakage over a broadband spectrum, we use a biaxial plate to substitute the positive A-plate, as shown in

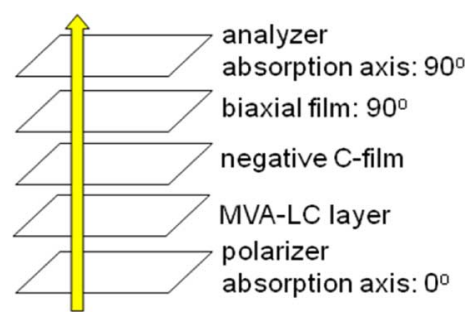

Fig. 4. MVA LC cell compensation configuration with one negative C-plate and one biaxial film.

Fig. 4. In this compensation scheme, LC cell still works under crossed linear polarizers. The absorption axes of polarizer and analyzer are $0^{\circ}$ and $90^{\circ}$, respectively. A layer of negative C-plate is located right above the MVA LC cell to compensate the phase retardation from $\mathrm{LC}$ at oblique viewing angle. Then a biaxial film is adopted mainly to reduce the light leakage from the crossed polarizers. It works approximately like a half wave plate. For the biaxial film employed here, $n_{x}>n_{z}>n_{y}$, we use $n_{x}$ direction to represent its orientation. It can be either parallel or perpendicular to the absorption axis of the bottom polarizer. The orientation can be selected according to the dispersion properties of the compensation films in order to obtain wide view over a broad spectral range. In our example, the dispersion of compensation films is much smaller than that of LC. So the biaxial film orientation is chosen to be $90^{\circ}$.

Fig. 5 illustrates the compensation principle for obtaining a broadband dark state. Fig. 5(a) shows the polarization tracing on Poincaré sphere at $\lambda=550 \mathrm{~nm}$. After passing through the polarizer, LC layer, and negative C-plate, the backlight polarization is changed from point $\mathrm{T}$ to $\mathrm{B}$ and then to $\mathrm{C}$. Here, the thickness of C-plate is carefully selected to make LC layer and negative C-plate work together as a thin positive C-plate. When the $N_{z}$ factor of biaxial film changing from 0 to 1 , in our configuration, the rotation axis on Poincare sphere changes from point $\mathrm{T}$ to $\mathrm{A}$. The arc length is dependent on its in-plane phase retardation value $d\left(n_{x}-n_{y}\right)$, where $d$ is the thickness and $\left(n_{x}-n_{y}\right)$ is the in-plane birefringence of the biaxial film. These parameters in our configuration are designed to ensure the polarization be moved from point $\mathrm{C}$ to $\mathrm{A}$, which is coincident to the absorption axis of the analyzer. In this way, a good dark state at $\lambda=550 \mathrm{~nm}$ can be achieved. Moreover, the light leakage at other wavelengths can also be suppressed significantly with small dispersion films. Let us take $\lambda=450 \mathrm{~nm}$ as an example as Fig. 5(b) shows, since C-plate has a weaker dispersion than LC layer, these two layers work as a positive C-plate with a larger phase retardation value at $\lambda=450 \mathrm{~nm}$ than at $550 \mathrm{~nm}$. Compared to the Poincaré sphere tracing at $\lambda=550 \mathrm{~nm}$, point $\mathrm{C}$ now is lower and further away from point $\mathrm{T}$. At the same time, the phase retardation of the consequent biaxial film is also larger. With a slightly smaller $N_{z}$ factor, the biaxial film can move the polarization from point $\mathrm{C}$ to $\mathrm{D}$ which is very close to point A, as shown in Fig. 5(b). Similarly, the polarization can also be moved to a point close to the polarization absorption axis of a longer wavelength, as shown in Fig. 5(d). The light leakage at $\lambda=450 \mathrm{~nm}$ and $650 \mathrm{~nm}$ after compensation is shown in Fig. 5(d) and (e), respectively. Compared with the conventional positive $\mathrm{A}$ and negative $\mathrm{C}$ compensation configuration, 


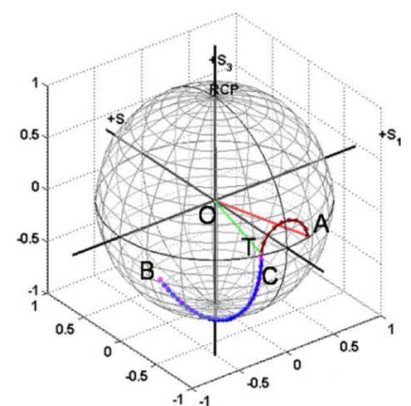

(a)

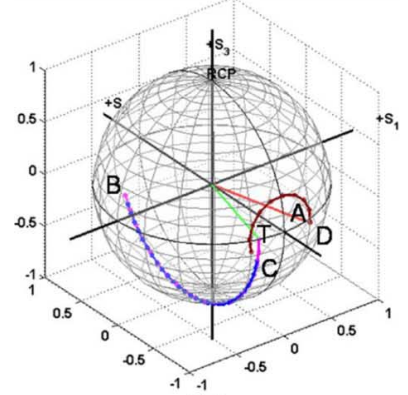

(b)

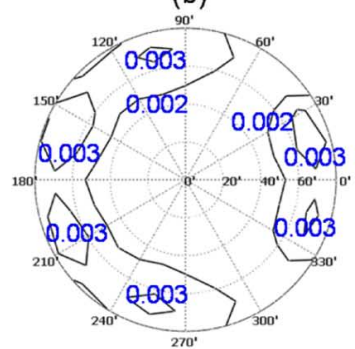

(c)

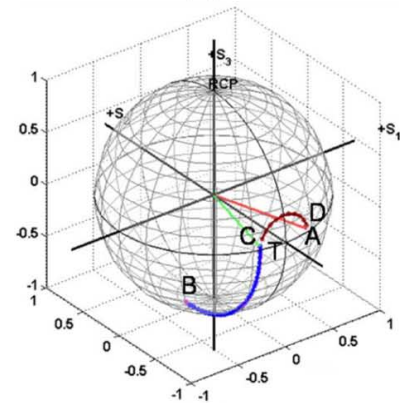

(d)

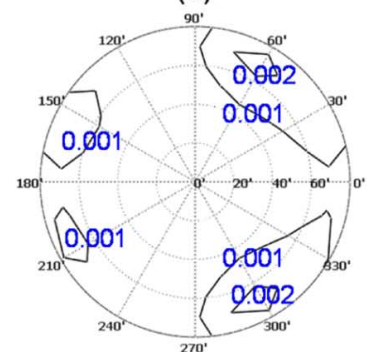

(e)

Fig. 5. Polarization state tracing on Poincaré sphere for the compensation scheme shown in Fig. 4 at (a) $\lambda=550 \mathrm{~nm}$; (b) $450 \mathrm{~nm}$; and (d) $650 \mathrm{~nm}$. The angular dependent light leakage is plotted in (c) for $450 \mathrm{~nm}$ and (e) for $650 \mathrm{~nm}$.

as shown in Fig. 3(b) and (d), our configuration suppresses the light leakage significantly at blue and red lights.

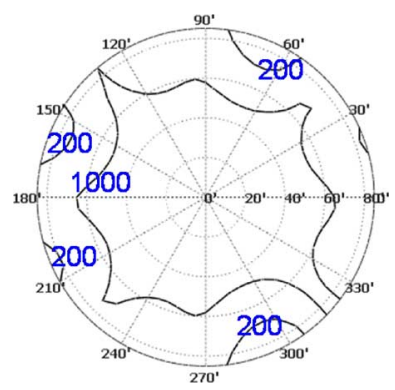

Fig. 6. Isocontrast contour of MVA-LCD at $\lambda=550 \mathrm{~nm}$ with the compensation scheme shown in Fig. 4.

\section{RESULTS}

In our calculation, the LC distribution is calculated by the finite element method [10] and the optical properties are calculated based on extended $2 \times 2$ Jones matrix methods [9], [11]. The phase compensation scheme is shown in Fig. 4. The LC cell together with two layers of compensation films are sandwiched between a pair of crossed linear polarizes. The LC mode considered is 4-domain VA with cell gap $d=4.5 \mu \mathrm{m}$. The LC used in our simulation is MLC6608 with parameters listed as follows: $K_{11}=16.7 \mathrm{pN}, K_{22}=7.3 \mathrm{pN}, K_{33}=18.1 \mathrm{pN}$, birefringence $\Delta n=0.0836$ at $\lambda=550 \mathrm{~nm}$, dielectric anisotropy $\Delta \varepsilon=-4.2$, and rotational viscosity $\gamma_{1}=186 \mathrm{mPa} \cdot \mathrm{s}$. The parameters for negative C-plate are $n_{e}=1.4925, n_{o}=1.5024$ at $\lambda=550 \mathrm{~nm}$. Its thickness is intentionally designed at 34.5 $\mu \mathrm{m}$ so that the total effect of the LC cell and negative C-plate is like a thin positive $\mathrm{C}$-plate. The parameters of the biaxial film at $\lambda=550 \mathrm{~nm}$ are optimized as follows: the in-plane birefringence $n_{x}-n_{y}=0.01, N_{z}$ factor $\left(n_{x}-n_{z}\right) /\left(n_{x}-n_{y}\right)=0.49$ and thickness $28.5 \mu \mathrm{m}$. With the compensation films designed as above, the viewing cone at $550 \mathrm{~nm}$ is expanded significantly. Meanwhile, the light leakage at other wavelengths can also be suppressed by a proper film dispersion design, which will be discussed later.

Fig. 6 shows the isocontrast contour plot at $\lambda=550 \mathrm{~nm}$ after compensation. The $\mathrm{CR} \geq 100: 1$ is over the entire viewing cone and $\mathrm{CR} \geq 200: 1$ is over $75^{\circ}$ viewing cone.

To obtain wide-viewing angle for a broadband backlight, the light leakage at red and blue should also be suppressed besides green. Thus, the dispersion properties of LC and compensation films need to be taken into consideration. Several polymeric films, such as PVA, exhibit a very weak dispersion characteristic because of the lacking of molecular conjugation [7]. Compensation films made from such a weak dispersive material would exhibit a much smaller dispersion than that of LC material consisting of a phenyl ring. Luckily we can take advantage of this dispersion difference to get self compensation for different wavelengths, which has been discussed in Section II. Within the small dispersion range, we optimized the birefringence of the negative C-plate and biaxial film at 450, 550, and $650 \mathrm{~nm}$ to get the least light leakage at these wavelengths. Fig. 7 shows the optimized results. 


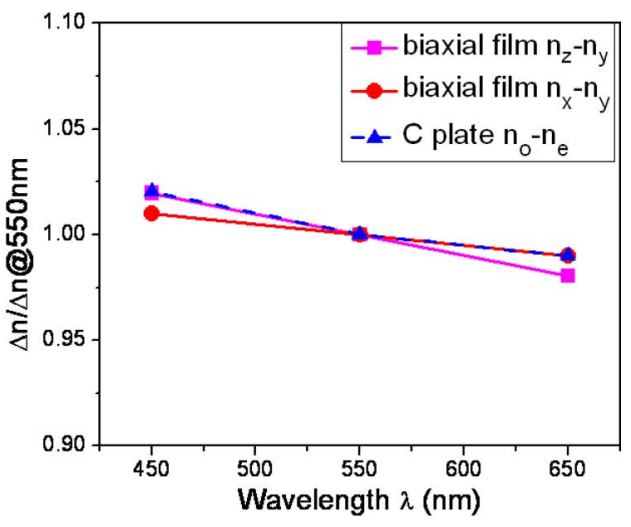

Fig. 7. Optimized birefringence dispersion of the compensation films.

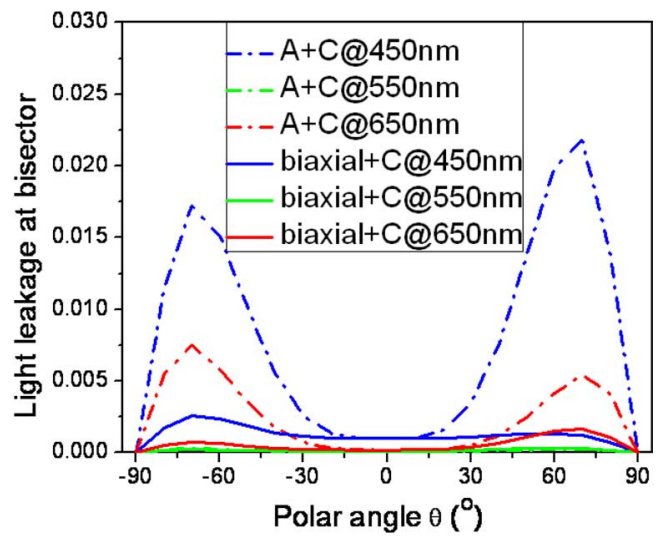

Fig. 8. Comparison of light leakages at bisectors between the conventional compensation scheme (Fig. 2(a)) and our proposed scheme (Fig. 4) at $\lambda=$ $450 \mathrm{~nm}, 550 \mathrm{~nm}$, and $650 \mathrm{~nm}$.

In our calculation, we use the birefringence dispersion model [12] to simulate LC's dispersion

$$
\Delta n=G \frac{\lambda^{2} \lambda^{* 2}}{\lambda^{2}-\lambda^{* 2}}
$$

where $G$ is a proportionality constant and $\lambda^{*}$ is the mean resonance wavelength. For compounds with a single phenyl ring, their $\lambda^{*}$ is around $210 \mathrm{~nm}$.

With the above designed parameters, the light leakage in the proposed configuration is greatly reduced. Fig. 8 shows the light leakage at the bisector direction of the two polarizers, where usually the maximum light leakage occurs so that the viewing angle is the worst. The solid lines represent the light leakage of the proposed compensation configuration with one negative $\mathrm{C}$-plate and one biaxial film, while the dash lines are for the conventional configuration with one negative C-plate and positive A-plate. The light leakage values are normalized to the maximum transmittance of two parallel polarizers. At $\lambda=550 \mathrm{~nm}$, both configurations show a very small light leakage at all viewing angle. However, there is still light leakage at $\lambda=450 \mathrm{~nm}$ and $\lambda=650 \mathrm{~nm}$ in the conventional configuration. By contrast, the light leakages at red and blue of our configuration are significantly reduced, which will lead to a much higher contrast ratio and wider viewing angle for a white light source employed.

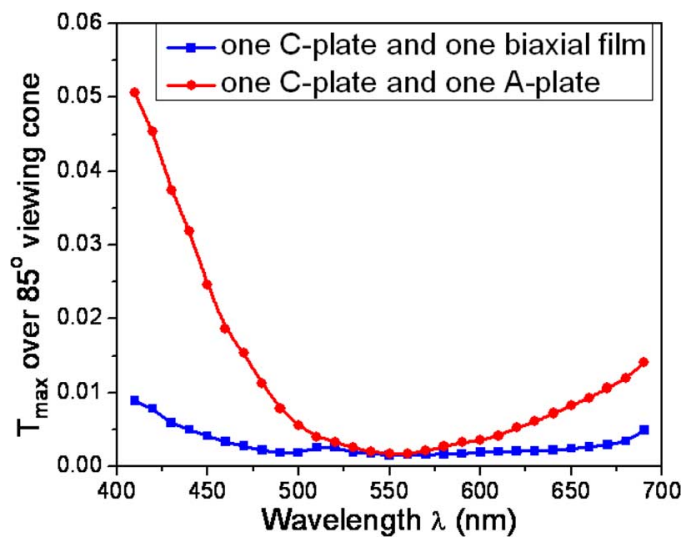

Fig. 9. Comparison of maximum light leakage over the whole viewing cone between (a) compensation scheme shown in Fig. 2(a) (red) and (b) Fig. 4 (blue).

Knowing the refractive index at the three optimized wavelengths, we can use the extended Cauchy equation [13] to obtain the refractive index in the entire visible spectra

$$
n=A+\frac{B}{\lambda^{2}}+\frac{C}{\lambda^{4}} .
$$

Once the refractive indices from $\sim 400 \mathrm{~nm}$ to $\sim 700 \mathrm{~nm}$ are obtained, we calculated the light leakage for the entire visible spectra. The global maximum light leakage over the entire viewing cone for each wavelength is plotted in Fig. 9, where red and blue curves represent the maximum light leakage for the configurations shown in Figs. 2(a) and 4, respectively. For the improved compensation scheme, the maximum light leakage is reduced by $\sim 6 X$ at $\lambda=450 \mathrm{~nm}$ and $\sim 4 X$ at $\lambda=650 \mathrm{~nm}$. The light leakage over the whole visible spectra is greatly suppressed in the proposed configuration as compared to the conventional one. With better dark state, the contrast ratio and viewing angle of a full-color LCD will be further improved.

\section{CONCLUSION}

We have proposed a new compensation configuration for MVA LCDs using a negative C-plate and a biaxial plate. With the designed phase retardation and using weak dispersive compensation films, the light leakage over entire visible spectra is reduced. The maximum light leakage over the entire viewing cone is reduced by $\sim 6 X$ at $\lambda=450 \mathrm{~nm}$ and $\sim 4 X$ at $650 \mathrm{~nm}$, as compared to the conventional compensation scheme. This approach will further enhance the contrast ratio, viewing angle, and color saturation of MVA LCDs.

\section{REFERENCES}

[1] J. Chen, K. H. Kim, J. J. Jyu, J. H. Souk, J. R. Kelly, and P. J. Bos, "Optimum film compensation modes for TN and VA LCDs," in SID Dig. Tech. Papers, 1998, vol. 29, pp. 315-318.

[2] X. Zhu, Z. Ge, and S. T. Wu, "Analytical solutions for uniaxial-film-compensated wide-viewing liquid crystal displays," $J$. Display. Technol., vol. 2, no. 1, pp. 2-20, Mar. 2006.

[3] Q. Hong, T. X. Wu, X. Zhu, R. Lu, and S. T. Wu, "Extraordinarily high-contrast and wide-view liquid-crystal displays," Appl. Phys. Lett., vol. 86, pp. 121107-121107, 2005

[4] I. Amimori, S. Suzuki, F. Obata, and C. Ruslim, "Deformed nanostructure of photo-induced biaxial cholesteric films and their application in VA-mode LCDs," J. SID, vol. 13, pp. 799-802, 2005. 
[5] T. Ishinabe, T. Miyashita, and T. Uchida, "Wide-viewing-angle polarizer with a large wavelength range," Jpn. J. Appl. Phys., vol. 41, pp. $4553-4558,2002$.

[6] S.-H. Ji, J.-M. Choi, and G.-D. Lee, "Optical compensation in a vertical alignment liquid crystal cell for elimination of the off-axis light leakage," in IMID/IDMC/Asia Display Dig., 2008, pp. 1580-1583.

[7] S. T. Wu, "Phase-matched compensation films for liquid crystal displays," Mat. Chem. Phys., vol. 42, pp. 163-168, 1995.

[8] K. Vermeirsch, A. D. Meyere, J. Fornier, and H. D. Villeeschouwer, "Viewing angle of liquid-crystal displays: Representation on the Poincaré sphere," Appl. Opt., vol. 38, pp. 2775-2786, 1999.

[9] Z. Ge, T. X. Wu, X. Zhu, and S. T. Wu, "Reflective liquid crystal displays with asymmetric incidence and exit angles," J. Opt. Soc. Amer. A., vol. 22, pp. 966-977, 2005.

[10] Z. Ge, T. X. Wu, R. Lu, X. Zhu, Q. Hong, and S. T. Wu, "Comprehensive three-dimensional dynamic modeling of liquid crystal devices using finite element method," J. Display Technol., vol. 1, no. 2, pp. 194-206, Dec. 2005.

[11] A. Lien, "Extended Jones matrix representation for the twisted nematic liquid-crystal display at oblique incidence," Appl. Phys. Lett., vol. 57, pp. 2767-2769, 1990

[12] S. T. Wu, "Birefringence dispersions of liquid crystals," Phys. Rev. A., vol. 33, pp. 1270-1274, 1986.

[13] J. Li and S. T. Wu, "Extended Cauchy equations for the refractive indices of liquid crystals," J. Appl. Phys., vol. 95, pp. 896-901, 2004.

Meizi Jiao received the B.S. degree in optics from Zhejiang University (ZJU), Hangzhou, China, in 2006, and is currently working toward the Ph.D. degree at the College of Optics and Photonics, University of Central Florida, Orlando.

Her current research interests include optical compensation films for wideview LCDs, and fast-response LC devices.
Zhibing Ge (S'02) received the B.S. degree in electrical engineering from Zhejiang University, Hangzhou, China, in 2002, and the M.S. and Ph.D. degrees in electrical engineering from the University of Central Florida, Orlando, in 2004 and 2007, respectively.

Since 2008, he has been with the College of Optics and Photonics at University of Central Florida as a research scientist. His research interests include novel liquid crystal displays and laser beam steering technologies. He has published 1 book chapter, over 30 journal papers, and 12 issued or pending patents in related area.

Dr. Ge is a recipient of the 2008 Otto Lehmann Award. Since May 2008, he has served as an associate editor for Journal of Society for Information Display $(J S I D)$ on the LCD division.

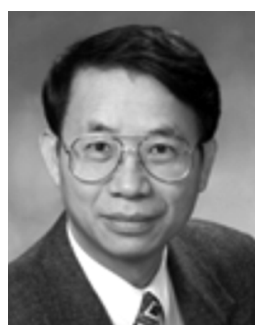

Shin-Tson Wu (M'98-SM'99-F'04) received the B.S. degree in physics from National Taiwan University, and the Ph.D. degree from the University of Southern California, Los Angeles.

$\mathrm{He}$ is a PREP professor at College of Optics and Photonics, University of Central Florida (UCF). His studies at UCF concentrate in liquid crystal displays, liquid crystal materials, optical communications, photonic crystal fibers, and bio-photonics. Prior to joining UCF in 2001, he worked at Hughes Research Laboratories, Malibu, CA, for 18 years. He has co-authored 5 books: Introduction to Flat Panel Displays (Wiley, 2008, with J. H. Lee and D. N. Liu), Fundamentals of Liquid Crystal Devices (Wiley, 2006, with D. K. Yang); Introduction to Microdisplays (Wiley, 2006, with D. Armitage and I. Underwood), Reflective Liquid Crystal Displays (Wiley, 2001, with D. K. Yang) and Optics and Nonlinear Optics of Liquid Crystals (World Scientific, 1993, with I. C. Khoo), as well as 6 book chapters, over 300 journal publications, and more than 55 issued patents.

Dr. Wu is a recipient of SPIE G. G. Stokes award and SID Jan Rajchman prize. $\mathrm{He}$ is a Fellow of the Society of Information Display (SID), Optical Society of America (OSA), and SPIE. 